\title{
Pattern Mining on How Organizational Tenure Affects the Psychological Capital of Employees Within the Hospitality and Tourism Industry: Linking Employees' Organizational Tenure With PsyCap
}

Donald Douglas Atsa'am, University of Agriculture, Makurdi, Nigeria

Ersin Kuset Bodur, Eastern Mediterranean University, Turkey

\begin{abstract}
Psychological capital (PsyCap) is a measure of the positive capabilities of an individual which consists of four components: hope, efficacy, resilience, and optimism. In this study, the pattern of the effect of organizational tenure on the PsyCap of employees in the hospitality and tourism industry was mined. The PsyCap and length of service data of a sample of 329 employees working in some organizations related to hospitality and tourism were collected. The odds ratio was deployed to measure the pattern and strength of association and the findings showed that employees who stay longer years in an organization are 3.6 times more likely to exhibit high PsyCap than those who stay shorter periods. With the positive pattern of this association, it implies that indiscriminate retrenchment of long-serving employees should be avoided to preserve PsyCap within the industry.
\end{abstract}

\section{KEYWORDS}

Employees' PsyCap, Hospitality and Tourism Employees, Odds Ratio, Organizational Tenure, Psychological Capital

\section{INTRODUCTION}

Psychological capital (PsyCap) is a measure of the positive capabilities of an individual which consists of four components: hope, efficacy, resilience, and optimism (Antunes, Caetano, \& Cunha, 2017; Luthans, Youssef, \& Avolio, 2007). Efficacy is the psychological quality of an individual that is reflected in their ability to carry out pending tasks diligently within a given time frame (Luthans \& Youssef, 2017; Stajkovic \& Luthans, 1998). Employees with positive efficacy usually source for resources to achieve set goals within time limits (Breevaart, Bakker, \& Demerouti, 2014). According to Kobau et al. (2011), optimism is a quality that associates positive events with personal and permanent 
causes, while interpreting negative events as external, temporary, and contextual. Optimistic individuals are flexible and pragmatic (Alarcon, Bowling, \& Khazon, 2013; Carver \& Scheier, 2002), focusing on positive outcomes while pursuing desired goals. Hope, in the opinion of (Snyder et al., 1991), is a state that motivates wishful thinking geared towards successfully achieving a desired goal. Resilience is the ability of an individual to recover from, or adapt to stress and adversity which could arise from family, workplace, financial or relationship problems (Lee \& Chu, 2016). Positive resilience enables individuals to persevere in unfavourable conditions (Tugade, Fredrickson, \& Barrett, 2004), which ultimately affect job performance and organizational outcomes. Collectively, these four components constitute psychological capital and have the positive effect on an individual, manifesting in dedication to duty, job performance, job satisfaction, and self-development (Avey, Reichard, Luthans, \& Mhatre, 2011). The commonly used measure of psychological capital is the Psychological Capital Questionnaire (PCQ) (Luthans, et al., 2007). The PCQ is a 6-point scale questionnaire, consisting of 24 items which employees utilize to rate themselves for the purpose of assessing their PsyCap. This measure has been validated by Luthans, Youssef-Morgan, and Avolio (2015) as an authentic tool, and has been adopted in many climes for psychological capital measurement (Antunes et al., 2017).

Several studies have been conducted to evaluate the relationship between psychological capital and some aspects of the work environment. One of such is the research by Simons and Buitendach (2013) undertaken to determine the relationship between employees' psychological capital and their commitment to the organization they work for. The study used the PCQ, demographic, work engagement and organizational commitment questionnaires to collect data on a sample of 106 call center employees in South Africa. The result showed that positive relationship exists between PsyCap, work engagement and organizational commitment. That is, employees with positive psychological capital are well committed and positively attached to the organization they work for. Another research by Leon-Perez, Antino, and Leon-Rubio (2016) reported that there exists a negative relationship between psychological capital and burnout on the one hand, and a positive relationship between psychological capital and quality of service on the other hand. The study was conducted on a sample of 798 workers in a Spanish vehicle safety and emissions inspection company. The psychological capital of the respondents was measured using the Psychological Capital Questionnaire, Quality of Service (QoS) was assessed using the QoS questionnaire, while burnout, also referred to as stress, was measured using the questionnaire developed by Shirom-Melamed (Leon-Perez et al., 2016). The research concluded that positive psychological capital among employees has high prospects in stress reduction and improved quality of service, which positively affects productivity. In a related research, Avey et al. (2010) investigated the relationship between positive psychological capital and employee's well-being over time. The study was conducted on 280 participants drawn from a Midwestern university. Their psychological capital was measured using the PCQ; psychological well-being was measured using the General Health Questionnaire and the Index of Psychological Well-Being (Avey et al., 2010). The research findings indicate that positive PsyCap enhances employee well-being, and psychological well-being has a proportionate effect on job satisfaction. Luo, Wang and Yi (2017) researched on the role psychological capital plays between corporate culture and job performance. The survey, conducted on 377 workers of a petrochemical company, revealed that corporate culture has a positive effect on the four components of psychological capital; and psychological capital has prospects to intermediate between corporate culture and job performance among employees. A study conducted by Durrah, Alhamoud, and Khan (2016) investigated the relationship between PsyCap and job performance on the one hand, and the mediating role of job satisfaction among PsyCap and job performance. The research utilized the Psychological Capital Questionnaire, job performance and job satisfaction questionnaires to collect data on 110 instructors from the Philadelphia University. The result show that positive PsyCap is positively related to job performance and job satisfaction mediates the relationship between PsyCap and job performance (Durrah et al., 2016). In another survey, Sun, Zhao, Yang, and Fan (2011) investigated how psychological capital impacts on job embeddedness and performance. A sample size of 1000 nurses in a university hospital in China provided the research 
data, and the findings indicate that a strong association exists between psychological capital, job embeddedness and work performance. The research holds that if the psychological capital of a nurse improves, their willingness to remain in the current work place as well as their job output will be substantially enhanced. A study by Atsa' am and Bodur (2019) investigated the relationship between psychological capital and educational qualifications among hospitality workers using odds ratios. The study reported that employees with higher qualifications are 2.6 more likely to be seen as having high psychological capital than those with lower qualifications.

The objective of this research is to extract the pattern of association between organizational tenure and PsyCap of employees in hospitality and tourism industry. It should be determined whether there exists any relationship between organizational tenure and PsyCap of employees serving in the hospitality and tourism industry. If such relationship exists, the pattern of the association (whether positive or negative) should be determined. As an individual works within an organization that offers hospitality or tourism services, is their psychological capital more likely to increase or decrease as years pass by? This research question is addressed by deploying the logistic regression to mine the pattern of association between organizational tenure and PsyCap using data obtained from a sample of hospitality and tourism employees.

\section{MATERIALS AND METHODS}

\section{Materials}

In this section, the main research tools deployed for experiments in this study are presented. These tools include the logistic regression and the odds ratio.

The logistic regression is given in Equation (1):

$\ln (P /(1-P))=b_{0}+b_{1} X_{i}$

where $b_{0}$ is the intercept, $b_{1}$ is a parameter estimate and $\mathrm{X}$ is a data value of the independent variable, $i=1, \ldots, n$ and $P /(1-P)$ is known as the odds (Grimes \& Schulz, 2008; Hosmer, Lemeshow Jr., \& Sturdivant, 2013; Kleinbaum \& Klei, 2010; Liu et al., 2014). From Equation (1), the value of P can be obtained as $P=\frac{\exp \left(b_{0}+b_{1} X\right)}{1+\exp \left(b_{0}+b_{1} X\right)}$. The odds is a measure of the probability of success of an event versus the probability of failure of that same event (Bland \& Altman, 2000; Sainani, 2001).

Definition 1: Let $P_{A}$ be the probability of favorable outcome in group A, and $P_{B}$ be the probability of favorable outcome in group B. Then, the odds ratio is computed by using the probabilities with respect to group A and group B as:

Odds Ratio $(O R)=\frac{P_{A} /\left(1-P_{A}\right)}{P_{B} /\left(1-P_{B}\right)}$

This definition is given in (Grimes \& Schulz, 2008; Kleinbaum \& Klein, 2010; Sheldrick, Chung, \& Jacobson, 2017). Unlike probability, the value of OR ranges from zero to infinity and is widely used as a means of mining the pattern (direction) and strength of association among groups (Bland \& Altman, 2000). When the odds ratio to be determined has to do with a dependence arrangement; that is, one variable is dependent upon another, a second definition of the OR is given (Sanaina, 2011). 
Table 1. Tabular definition of odds ratio

\begin{tabular}{|l|l|l|l|}
\hline & $X=0$ & \multicolumn{1}{|c|}{$X=1$} & \multicolumn{1}{c|}{ Total } \\
\hline$Y=0$ & $N_{00}$ & $N_{01}$ & $N_{00}+N_{01}$ \\
\hline$Y=1$ & $N_{10}$ & $N_{11}$ & $N_{10}+N_{11}$ \\
\hline Total & $N_{00}+N_{10}$ & $N_{01}+N_{11}$ & $N_{00}+N_{01}+N_{10}+N_{11}$ \\
\hline
\end{tabular}

Definition 2: Let $X$ be a binary independent variable, and let $Y$ be a binary dependent variable.

Then:

$N_{00}=$ observations with $X=0$ and $Y=0$

$N_{01}=$ observations with $X=0$ and $Y=1$

$N_{10}=$ observations with $X=1$ and $Y=0$

$N_{11}=$ observations with $X=1$ and $Y=1$

The odds ratio is defined by:

Odds Ratio $(O R)=\frac{N_{00} \times \mathrm{N}_{11}}{N_{01} \times \mathrm{N}_{10}}$

The value of $\mathrm{OR}$ is vital to understanding the pattern of an association. If $O R=1$, no relationship exists between $X$ and $Y$; If $O R<1, X$ is negatively related to $Y$; If $O R>1, X$ and $Y$ are positively related (Atsa'am, 2020; Hancock \& Kent, 2016; Szumilas, 2010; Tranmer \& Elliot, 2008). The Definition 2 of $O R$ can be represented in a $2 \times 2$ contingency table as shown in Table 1 .

Aside the Equation (3), the logistic regression framework can also be applied to evaluate the odds ratio between binary variables that exhibit a dependence structure (Tranmer \& Elliot, 2008). The logit model in Equation (1) can be written in terms of odds as:

$$
P_{i} /\left(1-P_{i}\right)=\exp \left(b_{0}+b_{1} X_{i}\right)
$$

Let it be recalled that the $X$ in Equations (4) is binary: 0 and 1. Now, when $X=0$; $O d d s=\exp \left(b_{0}+b_{1} \times 0\right)=\exp \left(\mathrm{b}_{0}\right)$ and when $X=1 ; \operatorname{Odds}=\exp \left(b_{0}+b_{1} \times 1\right)=\exp \left(b_{0}+b_{1}\right)$.

To evaluate whether OR results are reliable or not, any of these two methods can be used: confidence interval (CI) or $p$-values (Park, 2013; Szumilas, 2010). The confidence interval, usually represented by a lower and upper limit, symbolizes the uncertainty surrounding the OR estimate (Islam, 2013). The fact that a small part of a population is normally sampled for a study, the confidence interval gives an assurance that the actual population effect lies between the lower and upper confidence 
limits (Islam, 2013). In order to calculate the CI, the standard error of the log odds ratio (SE_logOR) is first evaluated as shown in Equation (5), (Lawson, 2004):

$$
S E_{-} \log \text { OR }=\sqrt{\frac{1}{N_{00}}+\frac{1}{N_{01}}+\frac{1}{N_{10}}+\frac{1}{N_{11}}}
$$

According to Bland and Altman (2000) and Islam (2013), the upper and lower 95\%CI are calculated as:

$\left.\begin{array}{l}\text { Upper Limit } 95 \% \mathrm{CI}=\exp \left[\log (O R)+1.96\left(S E_{-} \log O R\right)\right] \\ \text { Lower Limit } 95 \% \mathrm{CI}=\exp \left[\log (O R)-1.96\left(S E_{-} \log O R\right)\right]\end{array}\right\}$

A $p$-value of less than 0.05 confirms statistical significance of the OR estimate.

\section{METHODOLOGY}

\section{Data}

Psychological capital was assessed on a self-rate basis using the psychological capital questionnaire reported in Luthans et al. (2007) and adopted by Paek, Schuckert, Kim, and Lee (2015) for use in the hospitality industry. A total of 500 workers in some public and private organizations within the hospitality and tourism industry in Nigeria were supplied the PCQ in July/August 2017 to rate their own PsyCap. The workers provided responses on a 6-point scale, corresponding to $1=$ strongly disagree, $2=$ disagree, $3=$ somewhat disagree, $4=$ somewhat agree, $5=$ agree, and $6=$ strongly agree. Some sample questions that measure an individual's PsyCap include: "I feel confident helping to set targets/goals for myself" - [Efficacy]; "I always look on the bright side of things regarding my job" - [Optimism]; "I can think of many ways to reach my current goals" - [Hope]; "I feel I can handle many things at a time at my job" - [Resilience].

Besides the PsyCap data, information relating to how long an employee had served within the current organization was collected. This was termed "organizational tenure", and was categorized into four, namely; "less than one year", "1-3 years", "4-6 years", and "7 years and above". Each participant was required to choose one category that applied to their years of service in the organization. It should be noted that there are no extant laws regulating data collection for academic research purposes in Nigeria where the data for this research was collected. Relatedly, the academic institution to which the authors of this study belong does not require express ethics approval for research data collection. However, in accordance with international best practices, the following statements were included in the introductory part of the questionnaire: "Please, I would like you to rate your psychological capital on a six point scale, as well as your years in service in the current organization as indicated in the questionnaire. The answers you give shall be kept confidential and shall be used solely for this research. Please, do not disclose your name or any identifying information. Thank you." Impliedly, the consent of individual participants was obtained by virtue of questionnaire completion and submission.

After retrieving the questionnaires from the participants, the next task was to enforce data integrity. This was done by discarding questionnaires with incomplete responses and missing values. Out of the 500 questionnaires administered, a total of 171 were discarded for failing data integrity test and 329 were retained. A threshold of 65 out of 100 points was used to classify a respondent's PsyCap as "high psychological capital"; while workers with total scores from 0 to 64 were classified as "low psychological capital". When transformed to the 6-point Likert scale and taking into consideration that a few questions in the PCQ are in reverse scale, $65 \%$ is equivalent to 4 , which corresponds to 
Table 2. Demographic breakdown of participants

\begin{tabular}{|c|c|c|c|}
\hline Organization & Number of Employees & Gender & Sub-Total \\
\hline $\begin{array}{l}\text { 4-star and 5-star Hotels } \\
\text { Beach club } \\
\text { Ministry of Culture and } \\
\text { Tourism }\end{array}$ & $\begin{array}{l}150 \\
77 \\
102\end{array}$ & $\begin{array}{l}\text { Male } \\
\text { Female }\end{array}$ & $\begin{array}{l}222 \\
107\end{array}$ \\
\hline Age & Sub-Total & Cadre & Sub-Total \\
\hline $\begin{array}{l}18-41 \\
42 \text { and above }\end{array}$ & $\begin{array}{l}300 \\
29\end{array}$ & $\begin{array}{l}\text { Lower } \\
\text { Middle } \\
\text { Top }\end{array}$ & $\begin{array}{l}212 \\
96 \\
21\end{array}$ \\
\hline Tenure (in Years) & Sub-Total & & \\
\hline $\begin{array}{l}<1 \\
1-3 \\
4-6 \\
7 \text { and above }\end{array}$ & $\begin{array}{l}0 \\
255 \\
73 \\
1\end{array}$ & & \\
\hline
\end{tabular}

"somewhat agree". For this reason, a total score of 65 and above was categorized as high PsyCap. Workers that selected the "less than one year" or the "1-3 years" option in the organizational tenure categories were classified as having "short organizational tenure". Accordingly, workers who chose the 4-6 years or 7 years and above options were classified as long organizational tenure. In other words, for purposes of this study, short organizational tenure spans 0-3 years, while long organizational tenure spans 4 years and above. The internal consistency of the PsyCap and organizational tenure measures were evaluated using Cronbach's alpha and each yielded $\alpha=0.86$. Demographic breakdown of the participants is presented in Table 2.

\section{Experiments}

Two binary variables were defined for the experimental dataset as OrgTenure and PsyCapital; where OrgTenure is an independent variable and PsyCapital is the dependent variable. OrgTenure and PsyCapital variables are represented by $X_{\text {OrgTenure }}$ and $Y_{\text {PsyCapital }}$, respectively. Each of the variables can take only 0 and 1 ; where 0 represents low psychological capital as well as short organizational tenure, and 1 represents high psychological capital as well as long organizational tenure.

\section{Evaluation of the Odds Ratio}

For the specific purpose of this study, Definition 2 was modified as follows:

$$
\begin{aligned}
& N_{00}=\text { employees with } X_{\text {OrgTenure }}=0 \text { and } Y_{\text {PsyCapital }}=0 \\
& N_{01}=\text { employees with } X_{\text {OrgTenure }}=0 \text { and } Y_{\text {PsyCapital }}=1 \\
& N_{10}=\text { employees with } X_{\text {OrgTenure }}=1 \text { and } Y_{\text {PsyCapital }}=0 \\
& N_{11}=\text { employees with } X_{\text {OrgTenure }}=1 \text { and } Y_{\text {PsyCapital }}=1
\end{aligned}
$$

The $2 \times 2$ contingency table presented in Table 3 was produced when this definition was applied on the experimental dataset.

By applying the OR formula in Equation (3) to Table 3, the following result was obtained: 
Table 3. Cross tabulation of dataset binary variables

\begin{tabular}{|l|l|l|l|}
\hline & $X_{\text {OrgTenure }}=\operatorname{Short(0)}$ & $X_{\text {OrgTenure }}=\operatorname{Long}(1)$ & Total \\
\hline$Y_{\text {PsyCapital }}=$ Low(0) & 62 & 6 & 68 \\
\hline$Y_{\text {PsyCapital }}=$ High (1) & 193 & 68 & 261 \\
\hline Total & 255 & 74 & 329 \\
\hline
\end{tabular}

$O R=\frac{62 \times 68}{193 \times 6} \approx 3.6$

The OR computed using Equation (3) simply measures the association between two variables, regardless whether one is dependent and the other is independent. When the dependent structure is involved such as the case in this study where PsyCapital is dependent and OrgTenure is independent, the logistic regression framework is applied (Tranmer \& Elliot, 2008).

\section{Computations Using Logistic Regression Framework}

The logistic regression model was fit on the dataset, using the PsyCapital and OrgTenure variables, producing the estimates shown in Table 4.

From Table 4, the logit model was obtained as:

$\ln (P /(1-P))=1.1356+1.2922 X_{\text {OrgTenure }}$

By writing Equation (8) in terms of the odds, Equation (9) was obtained:

$P /(1-P)=\exp \left(1.1356+1.2922 X_{\text {OrgTenure }}\right)$

From Equation (9), when $X_{\text {OrgTenure }}=0$, then:

Odds $=\exp (1.1356+1.2922 \times 0)=\exp (1.1356)=3.113$

When $X_{\text {OrgTenure }}=1$, then:

Table 4. Logistic regression model

\begin{tabular}{|l|l|l|l|l|}
\hline & \multicolumn{1}{|c|}{ Estimate } & \multicolumn{1}{|c|}{ Std. Error } & \multicolumn{1}{|c|}{$\mathbf{z}$ Value } & \multicolumn{1}{c|}{$\operatorname{Pr}(>\mid \mathbf{z l})$} \\
\hline Intercept & 1.1356 & 0.1460 & 7.779 & $7.32 \mathrm{E}-15$ \\
\hline$X_{\text {OrgTenure }}$ & 1.2922 & 0.4502 & 2.870 & 0.0041 \\
\hline
\end{tabular}


The results in Equations (10) and (11) produced the odds ratio as:

$$
O R=\frac{11.334}{3.113} \approx 3.6
$$

\section{Odds Ratio Validation}

The results of the OR were validated using confidence interval and $p$-value. The Equation (5) was applied on Table 3 to calculate the standard error of the log odds ratio, and the following result was obtained:

$$
\text { SE_logOR }=\sqrt{\frac{1}{62}+\frac{1}{6}+\frac{1}{192}+\frac{1}{68}}=\sqrt{0.2017}=0.4502
$$

By using Equation (6), the 95\% upper and lower confidence limits were evaluated as follows:

$$
\left.\begin{array}{l}
\text { Upper Limit 95\%CI }=\exp (1.2922+1.96(0.4502))=\exp (2.1746)=8.7987 \\
\text { Lower Limit 95\%CI }=\exp (1.2922-1.96(0.4502)=\exp (0.4098)=1.5070
\end{array}\right\}
$$

Therefore, the $95 \% \mathrm{CI}$ is from 1.5070 to 8.7987 as shown in Equation (14). This confidence interval produced a $p$-value as $0.004<0.05$.

\section{RESULTS AND DISCUSSION}

The odds that an employee with short organizational tenure, $(X=0)$, will have high psychological capital is 3.11, while the odds that an employee with long organizational tenure, $(X=1)$, will have high psychological capital is 11.33. These results are presented in Equations (10) and (11). Even before computing the odds ratios, it is immediately apparent that the odds of a PsyCap improvement are in favour of employees with long organizational tenure. Interpreting the odds in terms of odds ratios, a value of 3.6 is obtained as shown in Equation (12). The implication of this result is that, an employee with long organizational tenure is 3.6 times more likely than an employee with short organizational tenure to be seen as having high psychological capital. In other words, employees seen as having high PsyCap are 3.6 times more likely to have stayed longer periods in their current organization than shorter periods. This proves that there is a positive pattern in the association between organizational tenure and psychological capital within the hospitality and tourism industry. That is, as an employee's organizational tenure increases, their psychological capital is 3.6 more likely to improve than decline.

The 95\% confidence interval (95\% CI 1.5 to 8.8) obtained in Equation (14) does not span the null value, 1 . Furthermore, the CI produced a $p$-value of $0.004<0.05$. This means that the result is reliable and statistically significant. This is an assurance that 95 out of every 100 times the odds ratio between employees psychological capital and organizational tenure is evaluated in the context of hospitality and tourism industry, using whatever population sample, the OR will fall between 1.5 
and 8.8. This is a valid research outcome given that the OR value of 3.6 obtained in this study falls within this range, more so that 1 is excluded from the confidence interval.

\section{CONCLUSION}

This study establishes that employees' psychological capital is positively related to their length of service within an organization in the hospitality and tourism sector. This finding is valuable in human resource management for the preservation of the psychological capital asset of an organization. Previous studies have shown that psychological capital is positively related to job performance (Durrah, et al., 2016; Luo et al., 2017), quality of service (Leon-Perez et al., 2016), job commitment (Simons \& Buitendach, 2013) and job embeddedness (Sun et al., 2011). This means that personnel managers ought to strive to retain workers with high psychological capital. It will, therefore, be counter-productive for organizations to retrench their employees with long organizational tenure as this research has established that psychological capital of an employee is associated with tenure of service. The most appropriate category of staff to consider during general retrenchment should be those employees with short organizational tenure as not much will be lost in terms of psychological capital. For emphasis, this research established that psychological capital of an employee improves over time as they serve within an organization in the hospitality and tourism sector. Therefore, it will be counterproductive for an enterprise to lay off employees that have served long tenures. It is recommended that human resource managers and other relevant stakeholders within the industry should develop strategies aimed at retaining or absorbing long-tenured employees within the hospitality and tourism sector. This would go a long way to harnessing the positive attributes associated with employees' psychological capital as guaranteed by long organizational tenure. The data used in this research was generated from three organizations within the hospitality and tourism industry namely; hotels, beach club, and Ministry of Culture and Tourism. Workers in areas such as aviation, academic tourism department, and game reserves were not covered. In future research, more organizations within the hospitality and tourism industry should be covered during data collection. 


\section{REFERENCES}

Alarcon, G. M., Bowling, N. A., \& Khazon, S. (2013). Great expectations: A meta-analytic examination of optimism and hope. Personality and Individual Differences, 54(7), 821-827. doi:10.1016/j.paid.2012.12.004

Antunes, A. C., Caetano, A., \& Cunha, M. P. (2017). Reliability and construct validity of the Purtuguese version of the psychological capital questionnaire. Psychological Reports, 120(3), 520-536. doi:10.1177/0033294116686742 PMID:28558609

Atsa'am, D. D. (2020). Feature selection algorithm using relative odds for data mining classification. In A. Haldorai, \& A. Ramu (Eds.), Big Data Analytics for Sustainable Computing (pp. 81-106). Hersey, PA: IGI Global. doi:10.4018/978-1-5225-9750-6.ch005

Atsa' am, D. D., \& Bodur, E. K. (2019). Knowledge mining on the association between psychological capital and educational qualifications among hospitality employees. Current Issues in Tourism. Advance online publication. doi:10.1080/13683500.2019.1597026

Avey, J. B., Luthans, F., Smith, R. M., \& Palmer, N. F. (2010). Impact of positive psychological capital on employee well-being over time. Journal of Occupational Health Psychology, 15(1), 17-28. doi:10.1037/ a0016998 PMID:20063956

Avey, J. B., Reichard, R. J., Luthans, F., \& Mhatre, K. H. (2011). Meta-analysis of the impact of positive psychological capital on employee attitudes, behaviors, and performance. Human Resource Development Quarterly, 22(2), 127-152. doi:10.1002/hrdq.20070

Bland, J. M., \& Altman, D. G. (2000). Statistics notes: The odds ratio. BMJ (Clinical Research Ed.), 320(7247), 1468. doi:10.1136/bmj.320.7247.1468 PMID:10827061

Breevaart, K., Bakker, A. B., \& Demerouti, E. (2014). Daily self-management and employee work engagement. Journal of Vocational Behavior, 84(1), 31-38. doi:10.1016/j.jvb.2013.11.002

Carver, C. S., \& Scheier, M. F. (2002). Control processes and self-organization as complementary principles underlying behavior. Personality and Social Psychology Review, 6(4), 304-315. doi:10.1207/ S15327957PSPR0604_05

Durrah, O., Alhamoud, A., \& Khan, K. (2016). Positive psychological capital and job performance: The mediating role of job satisfaction. International Scientific Research Journal, 72(7), 241-225. doi:10.21506/j.ponte.2016.7.17

Grimes, D. A., \& Schulz, K. F. (2018). Making sense of odds and odds ratios. Obstetrics and Gynecology, 111(2, Part 1), 423-426. doi:10.1097/01.AOG.0000297304.32187.5d PMID:18238982

Hancock, P., \& Kent, P. (2016). Interpretation of dichotomous outcomes: Risk, odds, risk ratios, odds ratios and number needed to treat. Journal of Physiotherapy, 62(3), 172-174. doi:10.1016/j.jphys.2016.02.016 PMID:27320830

Hosmer, D. W., Lemeshow, S. Jr, \& Sturdivant, R. X. (2013). Applied logistic regression (3rd ed.). Wiley. doi:10.1002/9781118548387

Islam, N. (2013). Symmetry of Odds Ratio. International Journal of Physical and Social Sciences, 3, 580-582.

Kleinbaum, D. G., \& Klein, M. (2010). Logistic regression: A self learning text (3rd ed.). Springer., doi:10.1007/978-1-4419-1742-3

Kobau, R., Seligman, M. E., Peterson, C., Diener, E., Zack, M. M., Chapman, D., \& Thompson, W. (2011). Mental health promotion in public health: Perspectives and strategies from positive psychology. American Journal of Public Health, 108(8), e1-e9. doi:10.2105/AJPH.2010.300083 PMID:21680918

Lawson, R. (2004). Small sample confidence intervals for the odds ratio. Communications in Statistics. Simulation and Computation, 33(4), 1095-1113. doi:10.1081/SAC-200040691

Lee, C., \& Chu, K. (2016). Understanding the effect of positive psychological capital on hospitality interns creativity for role performance. International Journal of Organizational Innovation, 8, 213-222. 
Leon-Perez, J. M., Antino, M., \& Leon-Rubio, J. M. (2016). The role of psychological capital and intragroup conflict on employees burnout and quality of service: A multilevel approach. Frontiers in Psychology, 7, 1-11. doi:10.3389/fpsyg.2016.01755 PMID:27895601

Liu, D., Li, T., \& Liang, D. (2014). Incorporating logistic regression to decision-theoretic rough sets for classifications. International Journal of Approximate Reasoning, 55(1), 197-210. doi:10.1016/j.ijar.2013.02.013

Luo, H., Wang, Y., \& Yi, L. (2017). The intermediate effect of psychological capital between culture and performance. The Human Factors and Ergonomics Society 2017 Annual Meeting. doi:10.1177/1541931213601704

Luthans, F., \& Youssef-Morgan, C. M. (2017). Psychological capital: An evidence-based positive approach. Annual Review of Organizational Psychology and Organizational Behavior, 4(1), 339-366. doi:10.1146/ annurev-orgpsych-032516-113324

Luthans, F., Youssef, C. M., \& Avolio, B. J. (2007). Psychological capital: Investing and developing positive organizational behavior. In D. L. Nelson \& C. L. Cooper (Eds.), Positive Organizational Behavior (pp. 9-24). SAGE Publications., doi:10.4135/9781446212752.n2

Luthans, F., Youssef-Morgan, C. M., \& Avolio, B. J. (2015). Psychological capital and beyond. Oxford University Press.

Paek, S., Schuckert, M., Kim, T. T., \& Lee, G. (2015). Why is hospitality employees psychological capital important? The effects of psychological capital on work engagement and employee morale. International Journal of Hospitality Management, 50, 9-26. doi:10.1016/j.jhm.2015.07.001

Park, H. (2013). An introduction to logistic regression: From basic concepts to interpretation with particular attention to nursing domain. Journal of Korean Academy of Nursing Administration, 43(2), 154-164. doi:10.4040/ jkan.2013.43.2.154 PMID:23703593

Rawlings, J. O., Pantula, S. G., \& Dickey, D. A. (1998). Applied regression analysis: A research tool (2nd ed.). Springer. doi: $10.1007 / \mathrm{b} 98890$

Sainana, K. L. (2011). Understanding odds ratios. American Academy of Physical Medicine and Rehabilitation, 3(3), 263-267. doi:10.1016/j.pmrj.2011.01.009 PMID:21402371

Sheldrick, R. C., Chung, P. J., \& Jacobson, R. M. (2017). Math matters: How misinterpretation of odds ratios and risk ratios may influence conclusions. Academic Pediatrics, 17(1), 1-3. doi:10.1016/j.acap.2016.10.008 PMID:27989279

Simons, J. C., \& Buitendach, J. H. (2013). Psychological capital, work engagement and organisational commitment amongst call centre employees in South Africa. SA Journal of Industrial Psychology, 39(2), 1-12. doi:10.4102/ sajip.v39i2.1071

Snyder, C. R., Harris, C., Anderson, J. R., Holleran, S. A., Irving, L. M., Sigmon, S. T., Yoshinobu, L., Gibb, J., Langelle, C., \& Harney, P. (1991). The will and the ways: Development and validation of an individualdifferences measure of hope. Journal of Personality and Social Psychology, 60(4), 570-585. doi:10.1037/00223514.60.4.570 PMID:2037968

Sperandei, S. (2014). Lessons in biostatistics: Understanding logistic regression analysis. Biochemia Medica, 24, 12-18. doi:10.11613/BM.2014.003 PMID:24627710

Stajkovic, A., \& Luthans, F. (1998). Self-efficacy and work-related performance: A meta-analysis. Psychological Bulletin, 24(2), 240-261. doi:10.1037/0033-2909.124.2.240

Stoltzfus, J. C. (2011). Logistic regression: A brief primer. Academic Emergency Medicine, 18(10), 1099-1104. doi:10.1111/j.1553-2712.2011.01185.x PMID:21996075

Sun, T., Zhao, X. W., Yang, L. B., \& Fan, L. H. (2012). The impact of psychological capital on job embeddedness and job performance among nurses: A structural equation approach. Journal of Advanced Nursing, 68(1), 69-79. doi:10.1111/j.1365-2648.2011.05715.x PMID:21645045

Szumilas, M. (2010). Explaining odds ratios. Journal of the Canadian Academy of Child and Adolescent Psychiatry, 19, 227-229. PMID:20842279 
Tranmer, M., \& Elliot, M. (2008). Binary logistic regression. The Cathie Marsh Centre for Census and Survey Research, Manchester, UK. Retrieved September 30, 2018, from http://hummedia.manchester.ac.uk/institutes/ cmist/archivepublications/working-papers/2008/2008-20-binary-logistic-regression.pdf

Tugade, M. M., Fredrickson, B. L., \& Barrett, L. F. (2004). Psychological resilience and positive emotional granularity: Examining the benefits of positive emotions on coping and health. Journal of Personality, 72(6), 1161-1190. doi:10.1111/j.1467-6494.2004.00294.x PMID:15509280

Donald Douglas Atsa'am is currently a Computer Science lecturer with the University of Agriculture, Makurdi, Nigeria. He holds a Ph.D. in Applied Mathematics and Computer Science from the Eastern Mediterranean University, Famagusta, North Cyprus. His research interests are in Data Mining and Knowledge Discovery, Computational Intelligence, and Machine Learning. Donald is a Certified Information Systems Auditor with several years of experience in Systems Audit and Control.

Ersin Kuset Bodur is a lecturer with the Department of Mathematics, Eastern Mediterranean University, Famagusta, North Cyprus. Her research interests are in Machine Learning and Data Mining. 